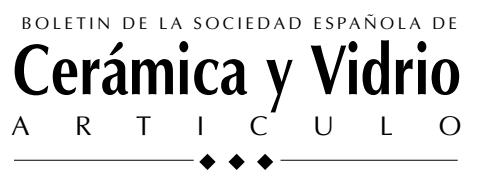

\title{
Preparación de tamices moleculares de carbono por CVD
}

\author{
R. MANSO ${ }^{1}$, J.A. PAJARES ${ }^{1}$, A. ALBINIAK ${ }^{2}$, E. BRONIEK ${ }^{2}$ Y T. SIEMIENIEWSKA ${ }^{2}$ \\ ${ }^{1}$ Instituto Nacional del Carbón, CSIC, Apartado 73, 33080 Oviedo, España \\ ${ }^{2}$ Institute of Chemistry and Technology of Petroleum and Coal, ul. Gdanska 7/9, 50-344 Wroclaw, Poland
}

\begin{abstract}
Se han preparado tamices moleculares de carbono (CMS) mediante el depósito de átomos de carbono, por pirólisis de benceno, sobre la superficie de carbones activados. La pirólisis de benceno a temperaturas comprendidas entre $650-850{ }^{\circ} \mathrm{C}$ genera el cierre progresivo de los microporos debido a la creación de constricciones en la red microporosa que limitan la accesibilidad de determinadas moléculas. El uso de temperaturas superiores a la temperatura de carbonización del precursor (850 ${ }^{\circ} \mathrm{C}$ ) introduce complicaciones debido a la descomposición y sinterización parcial del sólido. Flujos bajos de nitrógeno (30 mL $\mathrm{min}^{-1}$ ) con alto contenido en benceno $(13 \%$ ) producen un depósito homogéneo a lo largo de las paredes y los materiales presentan distribuciones microporosas mas anchas. El depósito de carbono sobre carbones activados por pirólisis de benceno a temperaturas inferiores a $850{ }^{\circ} \mathrm{C}$, utilizando flujos relativamente altos $\left(150 \mathrm{~mL} \mathrm{~min}^{-1}\right)$ con baja concentración de benceno $(1$ $\%$ ) genera tamices con volúmenes de microporos alrededor de $0,25 \mathrm{~cm}^{3} \mathrm{~g}^{-1} \mathrm{y}$ anchuras de poro distribuidas en intervalos estrechos: inferiores a 0,33 nm, entre 0,33-0,41 nm y entre 0,41-0,54 nm. El porcentaje de quemado del carbón activado de partida tiene relativamente poca influencia sobre las propiedades del tamiz molecular, cuya textura microporosa guarda una estrecha relación con un volumen de microporos controlado por el grado de depósito de carbono.
\end{abstract}

Palabras clave: Tamiz molecular de carbono, depósito de carbono, CVD, pirólisis, benceno, calorimetría de inmersión

\section{Preparation of carbon molecular sieves by CVD}

Carbon molecular sieves (CMS) have been prepared by chemical vapour deposition (CVD) of carbon from the pyrolysis of benzene molecules on activated carbon surfaces. The pyrolysis of benzene at temperatures in the range $650-850{ }^{\circ} \mathrm{C}$ restricts the accessibility of the micropores due to the creation of constrictions on the microporous network. Temperatures higher than $850^{\circ} \mathrm{C}$ (temperature of carbonisation) add difficulties due to decomposition and sinterization processes.

Low flows of nitrogen $(30 \mathrm{~mL} \mathrm{~min}-1)$ and high benzene content $(13 \%)$ produce a more uniform carbon deposition and wider micropore size distributions. The CVD process on carbons activated to different burn-offs, using temperatures below $850{ }^{\circ} \mathrm{C}$, flows of $150 \mathrm{~mL} \mathrm{~min}^{-1}$ and benzene content of $1 \%$, gives rise to microporous materials which exhibit micropore volumes around $0,25 \mathrm{~cm}^{3} \mathrm{~g}^{-1}$ and narrow micropore size distributions: below 0,33 nm, between 0,33-0,41 nm or between $0,41-0,54 \mathrm{~nm}$. The burn-off of the activated carbon has a relative little influence on the textural properties of the CMS that mainly depend on the degree of filling originated by the carbon deposition.

Key words: Carbon molecular sieve, carbon vapour deposition, CVD, pyrolysis, benzene, immersion calorimetry

\section{INTRODUCCIÓN}

Los tamices moleculares de carbono (CMS, de Carbon Molecular Sieves) son materiales carbonosos, microporosos y amorfos, con anchuras de poro similares a las dimensiones de moléculas pequeñas y distribuídas en un margen estrecho. Industrialmente están compitiendo y sustituyendo a las zeolitas como adsorbentes para la separación de oxígeno/nitrógeno del aire a temperatura ambiente. Diversas firmas comerciales los producen a nivel industrial - Carbo-Tech en Alemania, Takeda Chemical Company en Japón, Calgon Corporation en USA - siguiendo métodos basados en la apertura de materiales carbonosos mediante carbonización y activación, y un posterior cierre y control de los microporos por depósito de carbono mediante la pirólisis de hidrocarburos (1).

Poco se ha publicado acerca de las condiciones de prepara- ción y de su influencia en las propiedades de los materiales obtenidos. Lizzio (2) en 1993 realizó estudios sobre el efecto del depósito de carbono por pirólisis de metano sobre carbonizados. Verma (3) utiliza corrientes de propileno, Cabrera (4) piroliza isobutileno, Gaffney (5) 1, 3, 5 trimetilciclohexano... Mochida (6) llega a buenos resultados mediante la pirólisis de benceno y ciclohexano sobre fibras de carbono activadas. En este contexto, en este trabajo se presenta un estudio realizado sobre el depósito químico de carbono en fase vapor (CVD, de Chemical Vapour Deposition) mediante la pirólisis de benceno sobre carbones activados y la influencia de algunas variables - condiciones de flujo, temperatura y grado de quemado del carbón activo de partida - sobre la textura microporosa de los tamices(7). 


\section{PARTE EXPERIMENTAL}

Se prepararon pastillas de un carbón bituminoso de alto contenido en materia volátil, Pumarabule, 39,5\% M.V., que se oxidaron en aire a $250^{\circ} \mathrm{C}$ durante 72 horas, se carbonizaron hasta $850{ }^{\circ} \mathrm{C}$ y se activaron con vapor de agua hasta porcentajes de quemado del 20 y $40 \%$. Posteriormente se trataron en una corriente de $\mathrm{N}_{2}$ y benceno, a temperaturas entre $650{ }^{\circ} \mathrm{C}-900$ ${ }^{\circ} \mathrm{C}$, provocando la pirólisis del hidrocarburo y el depósito de átomos de carbono sobre la estructura porosa. En el dispositivo experimental (7) se utilizaron flujos diferentes, de 30, 150 y $200 \mathrm{~mL} \mathrm{~min}^{-1}$, con porcentajes de benceno del 1, 5 y $13 \%$.

La caracterización textural de las muestras se realizó mediante picnometrías de helio (densidad real) y mercurio (densidad aparente), porosimetría de mercurio (porosidad > $7,5 \mathrm{~nm}$ ), adsorción de $\mathrm{CO}_{2}$ y benceno a $25^{\circ} \mathrm{C}$ y calorimetría de inmersión en líquidos de diferente diámetro molecular, $\sigma(8)$ : diclorometano $\left(\mathrm{CH}_{2} \mathrm{Cl}_{2}, \sigma=0,33 \mathrm{~nm}\right)$, benceno $\left(\mathrm{C}_{6} \mathrm{H}_{6}\right.$, $\sigma=0,42 \mathrm{~nm})$, ciclohexano $\left(\mathrm{C}_{6} \mathrm{H}_{12}, \sigma=0,54 \mathrm{~nm}\right)$, tetracloruro de carbono $\left(\mathrm{C} \mathrm{Cl}_{4}, \sigma=0,63 \mathrm{~nm}\right), \mathrm{CDDT}$ (cis, trans, trans $-1,5$, 9 - ciclododecatrieno, $\left.\mathrm{C}_{12} \mathrm{H}_{21}, \sigma=0,76 \mathrm{~nm}\right)$ y a-pineno $\left(\mathrm{C}_{10}\right.$ $\mathrm{H}_{16^{\prime}} \sigma=0,80 \mathrm{~nm}$ ).

Los análisis inmediato y elemental del carbón bituminoso se realizaron en aparatos LECO TGA-601, LECO CHN-600 y LECO SC-132 conforme a las correspondientes Normas ISO. La densidad real se determinó en un Autopicnómetro Micromeritics AccuPyc 1330. La porosidad superior a 7,5 nm y la densidad aparente se determinaron en un porosímetro de mercurio Carlo Erba 2000, siguiendo un protocolo adaptado a carbones activados. Las medidas de adsorción de benceno y dióxido de carbono se llevaron a cabo en un aparato gravimétrico de tipo McBain con espirales de cuarzo, utilizando un método estático $(9,10)$. Para las entalpías de inmersión se utilizó un microcalorímetro Tian-Calvet (ThermoMetric 2277 Thermal Activity Monitor) con termopilas que convierten el calor desprendido en una señal eléctrica. La entalpía de inmersión se obtiene de la integración de esta señal frente al tiempo. El aparato se calibró mediante efecto Joule, en todo caso siendo la tolerancia de la medida calorimétrica $<1 \%$ (7).

\section{RESULTADOS Y DISCUSIÓN}

\subsection{Influencia de las condiciones de la corriente.}

Datos sobre el incremento (\% en peso) del carbono depositado sobre Pumarabule activado al $20 \%$, cuando la pirólisis de benceno se efectuó a $725{ }^{\circ} \mathrm{C}$, utilizando diferentes flujos y porcentajes de benceno en la corriente, se recogen en la Figura 1. El depósito de carbono es rápido durante los primeros minutos del proceso, disminuyendo progresivamente hasta alcanzar un valor asintótico, entre un 8-10 \% en peso, conforme a las distintas condiciones de la corriente gaseosa. El depósito es más rápido cuanto mayor es el porcentaje de benceno en la corriente; la mayor diferencia de concentraciones dentro y fuera de los microporos hace que las moléculas de benceno difundan más fácilmente hacia el interior de la red microporosa, donde debido a las altas temperaturas y a la acción catalítica de la superficie carbonosa rompen permitiendo el depósito de los átomos de carbono. Para corrientes con el mismo porcentaje de benceno, el uso de flujos más bajos determina un depósito más lento.

Las entalpías de inmersión en diclorometano de las muestras preparadas bajo diferentes condiciones de la corriente

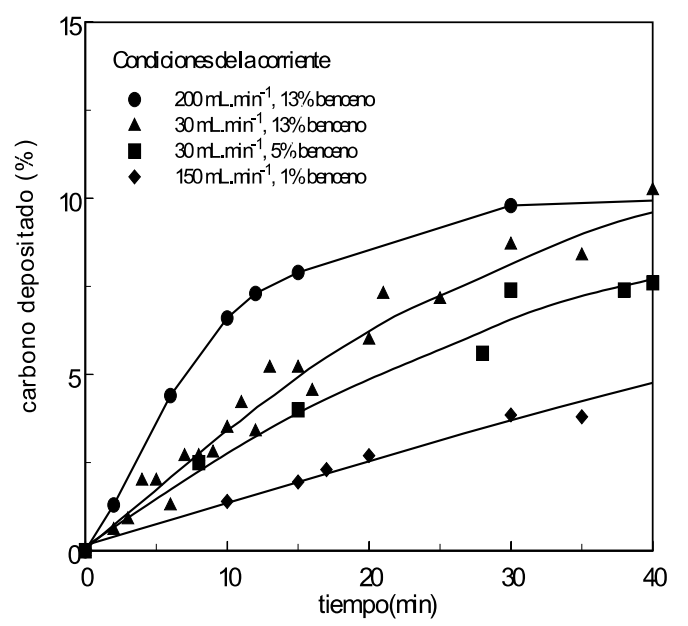

Figura 1. Incremento de peso del carbón Pumarabule activado al $20 \%$ por pirólisis de benceno a $725^{\circ} \mathrm{C}$

TABLA I. ENTALPÍAS DE INMERSIÓN EN LÍQUIDOS ORGÁNICOS DE MUESTRAS OBTENIDAS MEDIANTE DEPÓSITO DE UN $8 \%$ CARBONO SOBRE PUMARABUlE ACTIVADO AL $20 \%$ POR PIRÓLISIS DE BENCENO A $725^{\circ} \mathrm{C}$, BAJO DIFERENTES CONDICIONES DE LA CORRIENTE $\mathrm{N}_{2} /$ BENCENO.

\begin{tabular}{|c|c|c|c|c|c|}
\hline \multirow{2}{*}{$\begin{array}{c}\text { Flujo } \\
\left(\mathbf{m L}_{\mathbf{m i n}} \mathbf{- 1}^{-1}\right)\end{array}$} & $\begin{array}{c}\text { Benceno } \\
\mathbf{( \% )}\end{array}$ & \multicolumn{4}{|c|}{$-\Delta \mathbf{h i}\left(\mathrm{J.g}^{-\mathbf{1}}\right)$} \\
\cline { 3 - 6 } & $\mathbf{C H}_{\mathbf{2}} \mathbf{C l}_{\mathbf{2}}$ & $\mathbf{C}_{\mathbf{6}} \mathbf{H}_{\mathbf{6}}$ & $\mathbf{C}_{\mathbf{6}} \mathbf{H}_{\mathbf{1 2}}$ & $\mathbf{C D D T}$ \\
\hline $\mathbf{2 0 0}$ & $\mathbf{1 3 \%}$ & 86 & 16 & 8 & - \\
$\mathbf{3 0}$ & $\mathbf{1 3 \%}$ & 81 & 34 & 6 & - \\
$\mathbf{3 0}$ & $\mathbf{5 \%}$ & 79 & 12 & 6 & - \\
$\mathbf{1 5 0}$ & $\mathbf{1 \%}$ & 82 & 18 & 6 & 7 \\
\hline
\end{tabular}

(Tabla I) son similares indicando que en todas ellas el volumen de microporos accesible a esta molécula es semejante. El valor relativamente alto de la entalpía de inmersión en benceno ( $34 \mathrm{~J} \mathrm{~g}^{-1}$; Tabla I), para la muestra obtenida al utilizar un flujo de $30 \mathrm{~mL} \mathrm{~min}^{-1} \mathrm{y}$ un porcentaje de benceno en la corriente del $13 \%$, sugiere un proceso de depósito de carbono muy uniforme y regular sobre el conjunto de las paredes de microporos, siendo menos importante el efecto de formación de constricciones en la boca de los mismos. Tanto las concentraciones altas de benceno en la corriente como el uso de flujos bajos permite una mayor difusión hacia el interior de los microporos facilitando el depósito más uniforme a lo largo de sus paredes y, por consiguiente, dando lugar a materiales con distribuciones de anchura de microporos más anchas.

\subsection{Influencia de la temperatura de CVD}

Pumarabule activado al $20 \%$ fue sometido al proceso de CVD de carbono, bajo flujos de $150 \mathrm{~mL} \mathrm{~min}^{-1}$ de nitrógeno con un contenido en benceno del $1 \%$, utilizando distintas temperaturas de pirólisis de benceno entre $650-900{ }^{\circ} \mathrm{C}$ (Figura 2). La velocidad de depósito de carbono aumenta claramente con la temperatura utilizada en el intervalo comprendido entre 650$850{ }^{\circ} \mathrm{C}$; sin embargo a $900{ }^{\circ} \mathrm{C}$ la velocidad es inferior a la 


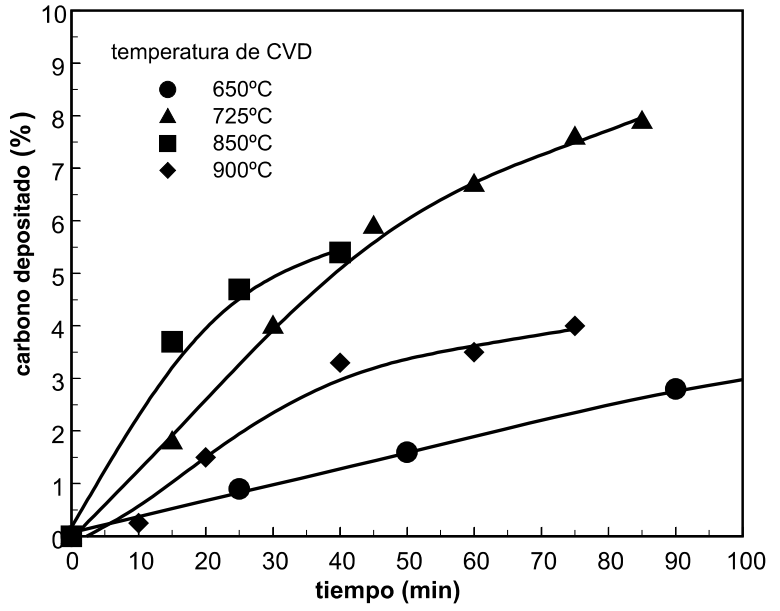

Figura 2. Cinética del depósito de carbono mediante pirólisis de benceno a diferentes temperaturas sobre el carbón Pumarabule activado al $20 \%$.

TABLA II. ENTALPÍAS DE INMERSIÓN DE MUESTRAS OBTENIDAS AL DEPOSITAR CARBONO A DISTINTAS TEMPERATURAS, UTILIZANDO UN FLUJO $\mathrm{N}_{2}$ / BENCENO DE 150 ML.MIN-1 CON UN $1 \%$ DE BENCENO, SOBRE Pumarabule activado al $20 \%$.

\begin{tabular}{|c|c|c|c|c|c|}
\hline T & Carbono depositado & \multicolumn{4}{|c|}{$-\Delta \mathbf{h i}\left(\mathrm{J.g}^{-\mathbf{1}}\right)$} \\
\hline$\left.{ }^{\circ} \mathbf{C}\right)$ & $\mathbf{( \% )}$ & $\mathbf{C H}_{\mathbf{2}} \mathbf{C l}_{\mathbf{2}}$ & $\mathbf{C}_{6} \mathbf{H}_{\mathbf{6}}$ & $\mathbf{C}_{6} \mathbf{H}_{12}$ & $\mathbf{C C l}_{4}$ \\
\hline $\mathbf{6 5 0}$ & $\mathbf{8}$ & 77 & 29 & 5 & 8 \\
$\mathbf{7 2 5}$ & $\mathbf{8}$ & 82 & 18 & 6 & 7 \\
$\mathbf{8 5 0}$ & $\mathbf{5}$ & 78 & 7 & 8 & 4 \\
$\mathbf{9 0 0}$ & $\mathbf{4}$ & 78 & 10 & 3 & - \\
\hline
\end{tabular}

encontrada a $725{ }^{\circ} \mathrm{C}$. El depósito químico de carbono a temperaturas comprendidas entre $650-850{ }^{\circ} \mathrm{C}$ sigue un mecanismo cinético de difusión interna (6), rompiendo las moléculas de benceno en su transporte hacia el interior de los microporos donde por adsorción química de los átomos de carbono se generan las constricciones que van a determinar las propiedades tamiz de los materiales finales. A temperaturas superiores a la de carbonización del precursor $\left(850{ }^{\circ} \mathrm{C}\right)$ se introducen complicaciones debido a que, paralelamente al depósito, se produce una pérdida de materia carbonosa y la sinterización del sólido; esto explicaría que la zona de saturación a $900{ }^{\circ} \mathrm{C}$ baje hasta $\approx 4 \%$ de carbono en peso.

Valores de entalpías de inmersión en los diferentes líquidos para muestras obtenidas por depósito de carbono a diferentes temperaturas sobre Pumarabule activado al $20 \%$ se recogen en la Tabla II. La entalpía de inmersión en benceno disminuye a medida que la temperatura de CVD aumenta en el intervalo $650-850{ }^{\circ} \mathrm{C}$. El mismo depósito de carbono a temperatura más alta sobre un carbón activado genera constricciones más estrechas que restringen el acceso a las moléculas, indicando que en general el proceso ha sido menos homogéneo y más dirigido hacia la boca de los microporos .

Cuanto más alta es la temperatura utilizada durante el depósito de un determinado porcentaje de carbono sobre un carbón activo, mayor es el cierre producido en la boca de los

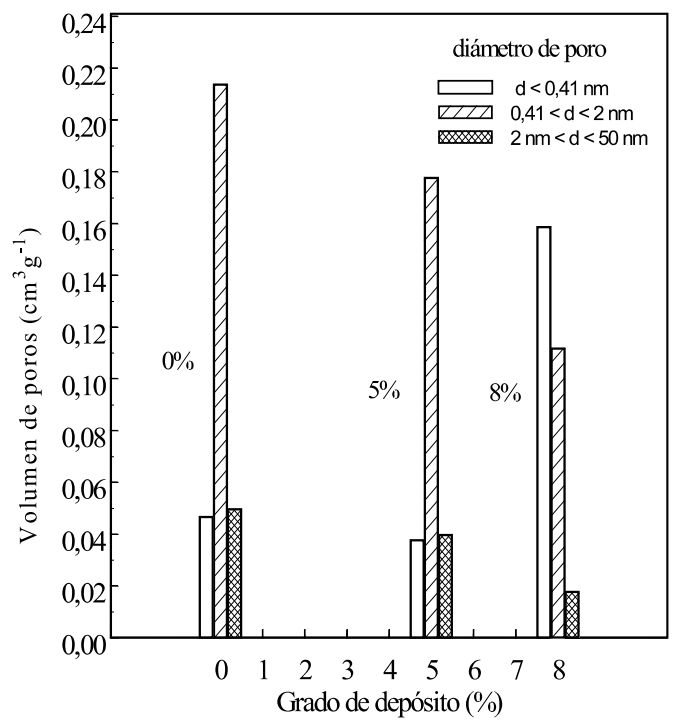

Figura 3. Volúmenes de meso y microporos obtenidos de las isotermas de $\mathrm{CO}_{2}$ y benceno a $25^{\circ} \mathrm{C}$ en los materiales preparados por CVD sobre Pumarabule activado al $20 \%$

TABla III. PARÁmetros teXtURALES DE MATERIALES PREPARAdOS MEDIANTE Depósito de CARbono SObRe Pumarabule aCtivado AL $20 \%$. (CONDICIONES DE LA CORRIENTE: 30 ML.MIN-1, $13 \%$ BENCENO)

\begin{tabular}{|c|c|c|c|c|c|c|}
\hline $\begin{array}{c}\text { CVD } \\
\mathbf{( \% )}\end{array}$ & $\begin{array}{c}\text { Densidad } \\
\text { real } \\
\left(\mathbf{g . c m}^{-3}\right)\end{array}$ & $\begin{array}{c}\text { Densidad } \\
\text { aparente } \\
\left(\mathbf{( g . c m}^{-3}\right)\end{array}$ & $\begin{array}{c}\text { V.Poros } \\
\text { total } \\
\left(\mathbf{c m}^{\mathbf{3}} \mathbf{g}^{-1}\right)\end{array}$ & $\begin{array}{c}\text { V.Poros } \\
\mathbf{d}>\mathbf{7 , 5} \mathbf{n m} \\
\left.\mathbf{( c m}^{\mathbf{3}} \mathbf{g}^{-1}\right)\end{array}$ & $\begin{array}{c}\text { V.Poros } \\
\mathbf{d}<7,5 \mathbf{n m} \\
\left(\mathbf{c m}^{\mathbf{3}} \mathbf{g}^{-1}\right)\end{array}$ & $\begin{array}{c}\text { Porosidad } \\
\mathbf{( \% )}\end{array}$ \\
\hline $\mathbf{0}$ & 2,09 & 1,02 & 0,50 & 0,25 & 0,25 & 51 \\
$\mathbf{3 , 6}$ & 2,04 & 1,07 & 0,44 & 0,24 & 0,20 & 47 \\
$\mathbf{8}$ & 2,01 & 1,09 & 0,42 & 0,23 & 0,19 & 46 \\
$\mathbf{1 0}$ & 1,99 & 1,13 & 0,38 & 0,22 & 0,17 & 43 \\
\hline
\end{tabular}

microporos. Las muestras preparadas a temperaturas superiores a la de carbonización no siguen esta tendencia; y la existencia de procesos paralelos al depósito de carbono afecta claramente la textura microporosa de la muestra obtenida.

\subsection{Influencia del grado de depósito de carbono}

A medida que aumenta el porcentaje de carbono depositado sobre los carbones activados se detecta un descenso progresivo de la densidad real y un aumento de la densidad aparente (Tabla III), lo que se traduce en una disminución del volumen total de poros. La fracción de poros con anchura inferior a 7,5 $\mathrm{nm}$ refleja un descenso paralelo.

El valor de los volúmenes y la distribución de microporos con diámetro inferior y superior a 0,41 nm, (correspondiente al diámetro de la molécula de benceno) así como la de mesoporos, se determinó por estudio de las isotermas de adsorción de $\mathrm{CO}_{2}$ y benceno (10); la Figura 3 muestra como el depósito progresivo de carbono sobre el carbón activo genera submicroporosidad debido a la creación de constricciones en los microporos, incrementando el volumen de microporos de anchura inferior a $0,41 \mathrm{~nm}$ a partir de la microporosidad más ancha. Los carbones de partida poseen un bajo porcentaje de 
mesoporosidad, que se reduce a medida que avanza el proceso de depósito, generándose microporos.

Los resultados de calorimetría de inmersión (Figura 4) permiten evaluar este cierre progresivo de la microporosidad a medida que avanza el proceso CVD. Tras el depósito del $5 \%$ de carbono sobre Pumarabule activado al $20 \%$ las entalpías de inmersión en diclorometano y benceno permanecen altas disminuyendo fundamentalmente las entalpías de inmersión en ciclohexano, tetracloruro de carbono y CDDT; el material obtenido presenta una pérdida de volumen del $17 \%$ ( Figura 3) y su microporosidad se encuentra distribuida fundamentalmente en la zona inferior a 0,54 nm (Figura 4).

Cuando el depósito de carbono alcanza un $8 \%$ las entalpías de inmersión reflejan el mayor cierre de las bocas de los microporos; el descenso de la entalpía de inmersión en benceno muestra el limitado acceso a esta molécula, mientras que la inmersión en diclorometano sigue dando un valor de entalpía alto, sugiriendo una importante contribución de los poros de anchura comprendida entre 0,33 y $0,41 \mathrm{~nm}$.

Tras el depósito del 10\% las muestras presentan una participación de microporos de diámetro superior a 0,33 nm prácticamente nula como indica la baja entalpía de inmersión en diclorometano $\left(9 \mathrm{~J} \mathrm{~g}^{-1}\right)$; la muestra sigue manteniendo el $64 \%$ de poros de diámetro inferior a 7,5 nm ( Tabla III), que corresponde a un volumen de poros de $0,16 \mathrm{~cm}^{3} \mathrm{~g}^{-1}$ (Figura 3), constituido fundamentalmente por microporos con diámetro inferior a $0,33 \mathrm{~nm}$ como se deduce del estudio comparativo de los resultados obtenidos de la calorimetría de inmersión y de las isotermas de adsorción.

Una representación gráfica de la distribución microporosa durante el cierre de poros hasta un $8 \%$ sobre Pumarabule activado al $20 \%$ se muestra en la Figura 5, donde queda muy clara la creación de la fracción de microporos entre 0,41-0,54 nm tras el depósito de un $5 \%$ de carbono; el sucesivo depósito posterior hasta un porcentaje del $8 \%$ fortalece las paredes dando lugar a un esqueleto de microporos de anchuras distribuidas preferentemente entre 0,33-0,41 nm.

\subsection{Influencia del porcentaje de quemado}

Carbones activados al 20 y $40 \%$ fueron sometidos a depósito químico de carbono por tratamiento a $725{ }^{\circ} \mathrm{C}$ con una corriente $\mathrm{N}_{2}$ / benceno de $150 \mathrm{~mL} \mathrm{m^{-1 }}$ y un porcentaje de benceno del $1 \%$. Entalpías de inmersión de algunos de los materiales obtenidos se representan en las Figuras 4 y 6 . Tamices con distribuciones de microporos inferiores a $0,54 \mathrm{~nm}$ se consiguen fácilmente mediante el depósito de un $5 \%$ sobre carbón activado al $20 \%$ (Figura 4) así como tras depositar un $15 \%$ sobre carbón activado al $40 \%$ (Figura 6). Un mayor grado de activación implica una mayor apertura de poros y por lo tanto para generar constricciones que originen entradas de anchura controlada es necesario un mayor depósito de carbono sobre la boca de los microporos. Las muestras obtenidas presentan similares distribuciones microporosas siendo la microporosidad accesible a cada líquido también del mismo orden, pudiendo concluir que a partir de carbones activados a diferentes porcentajes de quemado se consiguen muestras con microporosidad de características muy similares.

El depósito de porcentajes de carbono del 8 y $18 \%$ sobre carbones activados al 20 y $40 \%$, respectivamente, genera materiales con distribuciones de microporos entre 0,33-0,41

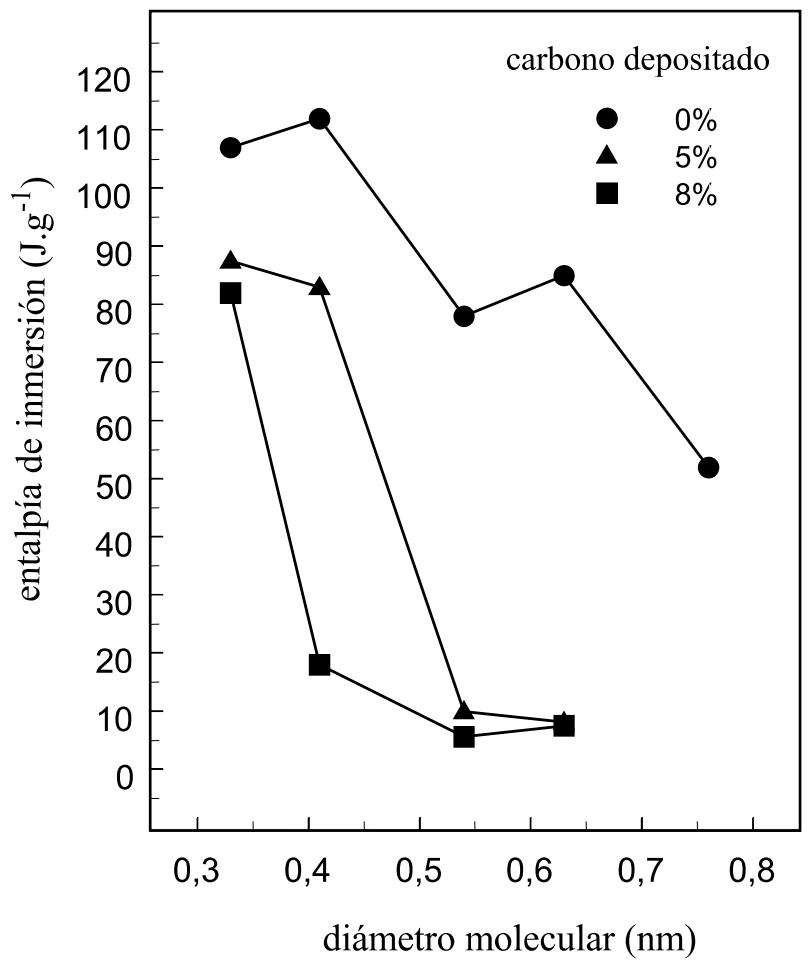

Figura 4. Entalpías de inmersión en líquidos orgánicos de diferente diámetro molecular de las muestras obtenidas mediante CVD de carbono sobre Pumarabule activado al $20 \%$

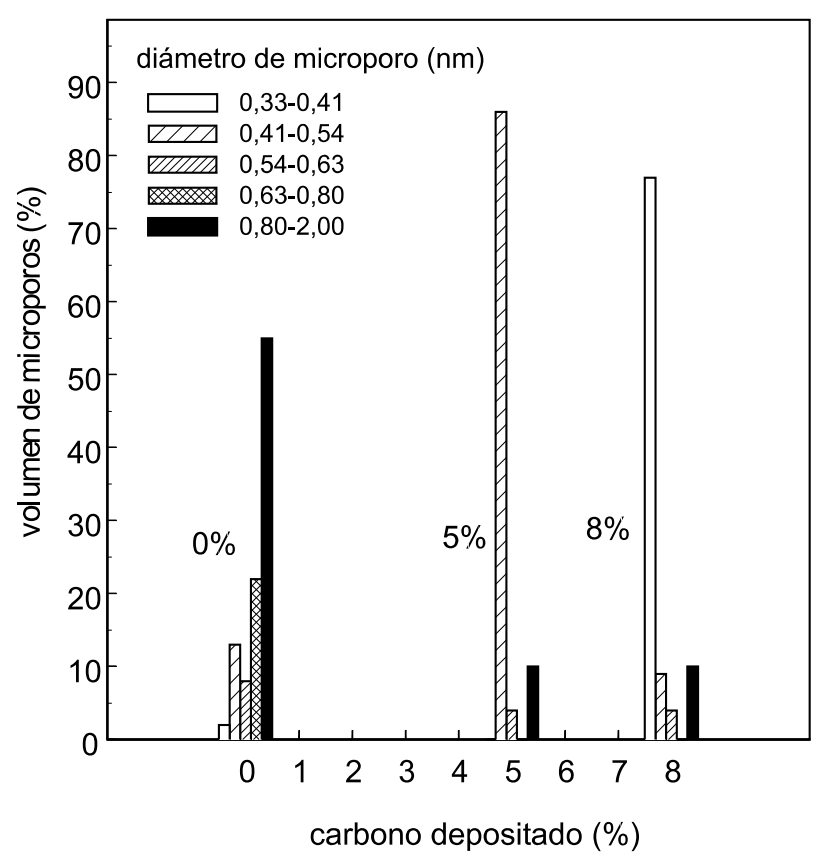

Figura 5. Distribución microporosa de materiales obtenidos por CVD de carbono sobre Pumarabule activado al $20 \%$. 


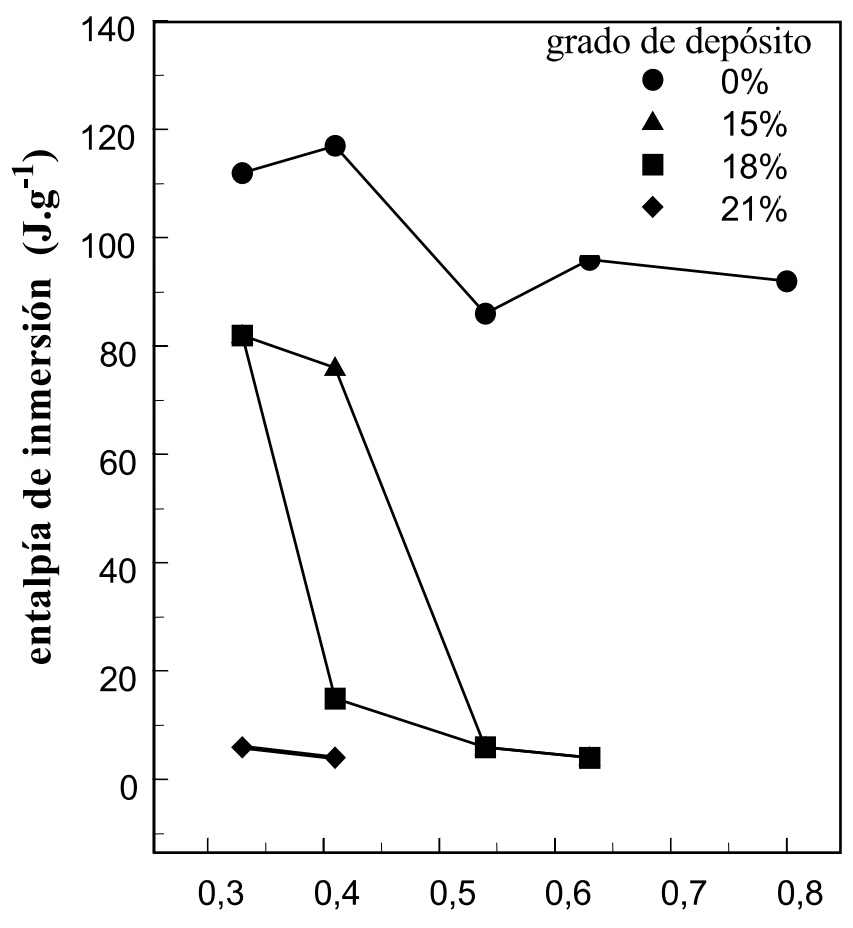

diámetro molecular (nm)

Figura 6. Entalpías de inmersión en líquidos de diferente diámetro molecular de las muestras obtenidas mediante CVD de carbono sobre Pumarabule activado al $40 \%$.

$\mathrm{nm}$. Las entalpías de inmersión en diclorometano se encuentran en torno a $80 \mathrm{~J} \mathrm{~g}^{-1}$, en benceno entre $15-18 \mathrm{~J} \mathrm{~g}^{-1}$, y en ciclohexano entre 6-8 $\mathrm{J} \mathrm{g}^{-1}$, por lo que se puede concluir que la microporosidad de las muestras obtenidas es muy similar.

Los resultados de la calorimetría de inmersión indican una influencia prácticamente despreciable del porcentaje de quemado del carbón activo de partida sobre la textura microporosa de los materiales obtenidos, que guarda una estrecha relación con un volumen de microporos controlado fundamentalmente por el grado de depósito de carbono.

\section{CONCLUSIONES}

La preparación de tamices moleculares por depósito químico de carbono a partir de corrientes gaseosas nitrógeno/benceno sobre carbones activados se controla favorablemente con flujos de corriente nitrógeno/benceno relativamente altos $\left(150 \mathrm{~mL} \mathrm{~min}^{-1}\right)$ y una baja concentración en benceno $(1 \%$ en volumen), que facilitan la formación de constricciones, y temperaturas entre $725-800{ }^{\circ} \mathrm{C}$, inferiores a la de carbonización, para evitar reacciones paralelas de descomposición y sinterización. Por el contrario, flujos bajos y contenidos de benceno elevados, dan lugar a depósitos de carbono muy uniformes a lo largo de la red porosa del carbón activo, con distribuciones de anchura de microporos más anchas.

Los tamices moleculares obtenidos presentan volúmenes de microporos de $0,20-0,25 \mathrm{~cm}^{3} \mathrm{~g}^{-1} \mathrm{y}$ distribuciones de tamaño de microporos muy estrechas: inferiores a $0,33 \mathrm{~nm}, 0,33-0,41 \mathrm{~nm}$ y 0,41-0,54 nm, conforme al porcentaje de carbono depositado.

\section{AGRADECIMIENTOS}

Los autores agradecen al MEC la financiación de este trabajo a través del proyecto DGICYT PB94-0012-C0301; al Ministerio de Asuntos Exteriores por la Acción Concertada Hispano-Polaca, 99PL0025, y al Comité Polaco para la Investigación Científica (KBN, Proyecto 3T0913 09614).

\section{BIBLIOGRAFÍA}

1. H. Juntgen, K. Knoblauch y K. Harder, “Carbon molecular sieves: production from coal and application in gas separation", Fuel 60, 817-822, 1981.

2 . A. A. Lizzio y M. Rostam-Abadi, "Production of carbon molecular sieves from Illinois coal", Fuel Processing Technology, 34, 97-103, 1993

3. S. K. Verma, "Development of molecular sieving properties in microporous carbon", Carbon 29, 793-803, 1991.

4. A.L. Cabrera y J.N. Armor, Air Products, "Modified carbon molecular sieve adsorbents", U.S. Patent No 5071450, 1991

5. T.R. Gaffney, T. S. Farris, A.L. Cabrera y J.N. Armor, Air Products, "Modified carbon molecular sieves for gas separation", U.S. Patent $N^{\circ}$ 5098880, 1992.

6 . Y. Kawabuchi, M. Kishimo, S. Kawaro, D. D. Whitehurst y I. Mochida, "Carbon deposition from benzene and cyclohexane onto active carbon fiber to control its pore size", Langmuir, 12, 4281-4285, 1996

7. R. Manso, "Preparación de tamices moleculares de carbono a partir de carbones minerales", Tesis doctoral, Universidad de Salamanca, 1999

8 . F. Krachenbuehl, F. Stoeckli, A. Addour, P. Ehrburger y J.B. Donnet, "The use of inmersion calorimetry in the determination of micropore distribution of carbons in the course of activation", Carbon, 24, 483-488, 1986

9.T.Siemieniewska, K. Tomkow, J.Kaczmarczyk, A.Albiniak, Y.Grillet y M.Francois, "Evaluation of microporosity in steam activated brown coal humic acid chars", en Characterization of Porous Solids II, F. Rodríguez Reinoso, J.Rouquerol, K.S.W. Sing y K.K. Unger Eds., Studies in Surface Science and Catalysis 62, p. 357-366. Elsevier, Amsterdam, 1991.

10. A. Albiniak, E. Broniek, M. Jasienko-Halat, A. Jankowska, J. Kaczmarczyk, T. Siemieniewska, R. Manso y J.A. Pajares, "Comparison of the porosity evaluation results based on inmersion calorimetry and gravimetric sorption measurements, for activated chars from a high volatile bituminous coal", Characterization of Porous Solids V, K.K. Unger, G.Kreysa y J.P.Baselt Eds., Studies in Surface Science and Catalysis 128, p. 653-662, Elsevier, Amsterdam, 2000

Recibido: 05.07.00 Aceptado: 05.07.00 